\section{State Estimation for Delayed Neural Networks}

\author{
Zidong Wang, Daniel W. C. Ho, and Xiaohui Liu
}

\begin{abstract}
In this letter, the state estimation problem is studied for neural networks with time-varying delays. The interconnection matrix and the activation functions are assumed to be norm-bounded. The problem addressed is to estimate the neuron states, through available output measurements, such that for all admissible time-delays, the dynamics of the estimation error is globally exponentially stable. An effective linear matrix inequality approach is developed to solve the neuron state estimation problem. In particular, we derive the conditions for the existence of the desired estimators for the delayed neural networks. We also parameterize the explicit expression of the set of desired estimators in terms of linear matrix inequalities (LMIs). Finally, it is shown that the main results can be easily extended to cope with the traditional stability analysis problem for delayed neural networks. Numerical examples are included to illustrate the applicability of the proposed design method.
\end{abstract}

Index Terms-Exponential stability, linear matrix inequalities (LMIs), neural networks, state estimation, time-delays.

\section{INTRODUCTION}

In 1983, Cohen and Grossberg published their seminal paper [5] on the global mathematical analysis of a class of general nonlinear cooperative-competitive neural networks, known as Cohen-Grossberg model. Since then, the mathematical properties of various neural networks, such as the stability, the attractivity and the oscillation, have been hot research topics that have drawn considerable attention, and a large amount of results have been available in the literature.

On the other hand, the dynamic behavior of many biological and artificial neural networks contains inherent time delays, which may cause oscillation and instability [1], [4], [12]. Delayed neural networks have been widely applied in many areas, such as signal and image processing, artificial intelligence, system identification, industrial automation, etc. In the past decade, the analysis of neural networks with time-delay has been a subject of great practical importance that has attracted a great deal of research interest. Up to now, most works on delayed neural networks have focused on the stability analysis problem for neural networks with constant or time-varying delays. Sufficient conditions, either delay-dependent or delay-independent, have been proposed to guarantee the asymptotical or exponential stability for the neural networks, see [1], [4], [9]-[12], [15], [17], and [18] for some recent results.

Since delayed neural networks have been considered as viable network models, the neuron state estimation problem becomes precursor for many applications. The main reason is that, in relatively large-scale neural networks, it is often the case that only partial information about the neuron states is available in the network outputs. Therefore, in order to utilize the neural networks, one often needs to estimate the neuron state through available measurement, and then use the estimated neuron

Manuscript received August 27, 2003; revised March 12, 2004. This work was supported in part by the Engineering and Physical Sciences Research Council (EPSRC) of the U.K. under Grant GR/S27658/01, in part by the Nuffield Foundation of the U.K. under Grant NAL/00630/G, in part by the RGC of Hong Kong SAR under Grant CityU/101103, and in part by the Alexander von Humboldt Foundation of Germany.

Z. Wang and X. Liu are with the Department of Information Systems and Computing, Brunel University, Uxbridge, Middlesex, UB8 3PH, U.K. (e-mail: Zidong.Wang@brunel.ac.uk).

D. W. C. Ho is with the Department of Mathematics, City University of Hong Kong, Kowloon, Hong Kong.

Digital Object Identifier 10.1109/TNN.2004.841813 state to achieve certain practical performances, such as system modeling, signal processing and control engineering. The state estimation problem for neural networks has received some research attention. In [6], an approach was developed to approximate the dynamic and static equations of stochastic nonlinear systems and to estimate state variables based on a radial basis function neural network. In [7], a method of estimating the online immeasurable states was proposed based on a recurrent neural network model of the system by using the extended Kalman filter. In [14], an adaptive state estimator was described by using techniques of optimization theory, the calculus of variations and gradient descent dynamics. So far, to the best of the authors' knowledge, though the stability analysis issue of delayed neural networks has been well studied, the important state estimation problem has not been fully investigated yet, and remains to be challenging. It is, therefore, our intention in this letter to deal with this problem, and provide a systematic design procedure of the desired state estimators.

In this letter, the state estimation problem is studied for neural networks with time-varying delays. The interconnection matrix and the activation functions are assumed to be norm-bounded. The problem addressed is to estimate the neuron states, through available output measurements, such that for all admissible time-delays, the dynamics of the estimation error is globally exponentially stable. An effective linear matrix inequality (LMI) approach is developed to solve the neuron state estimation problem. In particular, we derive the conditions for the existence of the desired estimators for the delayed neural networks. We also parameterize the explicit expression of the set of desired estimators, and show that the main results can be used to establish the stability criterion for a general class of delayed neural networks. Two numerical examples are used to demonstrate the usefulness of the proposed design methods.

The rest of the letter is arranged as follows. The state estimation problem is formulated in Section II for continuous delayed neural networks. In Section III, we give the main results that comprise the existence conditions and the explicit expression of the desired estimators. In Section IV, the results are extended for the stability analysis problem of delayed neural networks. Illustrative examples are provided in Section V, and some remarks are concluded in Section VI.

Notations: The notations are quite standard. Throughout this letter, $\mathbb{R}^{n}$ and $\mathbb{R}^{n \times m}$ denote, respectively, the $n$-dimensional Euclidean space and the set of all $n \times m$ real matrices. The superscript $T$ denotes matrix transposition and the notation $X \geq Y$ (respectively, $X>Y$ ) where $X$ and $Y$ are symmetric matrices, means that $X-Y$ is positive-semidefinite (respectively, positive-definite). $I_{n}$ is the $n \times n$ identity matrix. $|\cdot|$ is the Euclidean norm in $\mathbb{R}^{n}$. If $A$ is a matrix, denote by $\|A\|$ its operator norm, i.e., $\|A\|=\sup \{|A x|:|x|=1\}=\sqrt{\lambda_{\max }\left(A^{T} A\right)}$ where $\lambda_{\max }(\cdot)$ (respectively, $\lambda_{\min }(\cdot)$ ) means the largest (respectively, smallest) eigenvalue of $A . l_{2}[0, \infty]$ is the space of square integrable vector function. Denote by $L_{\mathcal{F}_{0}}^{p}\left([-h, 0] ; \mathbb{R}^{n}\right)$ the family of all $\mathcal{F}_{0}$-measurable $C\left([-h, 0] ; \mathbb{R}^{n}\right)$-valued variables $\xi=\{\xi(\theta):-h \leq \theta \leq 0\}$ such that $\sup _{-h<\theta<0}|\xi(\theta)|^{p}<\infty$. The shorthand $\operatorname{diag}\left\{\bar{M}_{1}, \bar{M}_{2}, \cdots, M_{N}\right\}$ denotes a block diagonal matrix with diagonal blocks being the matrices $M_{1}, M_{2}, \cdots, M_{N}$. Sometimes, the arguments of a function or a matrix will be omitted in the analysis when no confusion can arise.

\section{Problem Formulation AND PRELIMINARIES}

Consider the following delayed neural network with $n$ neurons:

$$
\dot{u}(t)=-A u(t)+W_{0} g(u(t))+W_{1} g(u(t-h(t)))+V
$$


where $u(t)=\left[u_{1}(t), u_{2}(t), \cdots, u_{n}(t)\right]^{T} \in \mathbb{R}^{n}$ is the state vector of the neural network, $A=\operatorname{diag}\left(a_{1}, a_{2}, \cdots, a_{n}\right)$ is a diagonal matrix with positive entries $a_{i}>0 . W_{0}=\left(w_{i j}^{0}\right)_{n \times n}$ and $W_{1}=\left(w_{i j}^{1}\right)_{n \times n}$ are the connection weight matrix and the delayed connection weight matrix, respectively. $g(u(t))=\left[g_{1}\left(u_{1}\right), g_{2}\left(u_{2}\right), \cdots, g_{n}\left(u_{n}\right)\right]^{T}$ denotes the neuron activation function with $g(0)=0$, and $V=\left[V_{1}, V_{2}, \cdots, V_{n}\right]^{T}$ is a constant vector. $h(t)$ denotes the time-varying bounded state delay satisfying

$$
0 \leq h(t) \leq h<\infty, \quad \dot{h}(t) \leq d<1
$$

where $h$ and $d$ are scalar constants.

Traditionally, when modeling a neural network, a typical assumption is that the activation functions are continuous, differentiable, monotonically increasing and bounded, such as the sigmoid-type of function. However, in many electronic circuits, the input-output functions of amplifiers may be neither monotonically increasing nor continuously differentiable. As discussed in [12] and [13], nonmonotonic functions can be more appropriate to describe the neuron activation in designing and implementing an artificial neural network. In this letter, we assume that the neuron activation function in $(1), g(\cdot)$, satisfies the following Lipschitz condition:

$$
|g(x)-g(y)| \leq|G(x-y)|
$$

where $G \in \mathbb{R}^{n \times n}$ is a known constant matrix. Hence, we do not need the activation functions to be monotonic and smooth. The type of activation functions in (3) is more general than the conventional sigmoid activation functions, and have been used in numerous papers [12].

In practice, it is often the case that the information about the neuron states are incomplete from the network measurements (outputs). That is, only partial information about the neuron states is available in the network measurements. On the other hand, the network measurements are subject to nonlinear disturbances. Therefore, our aim is to develop an efficient estimation algorithm in order to observe the neuron states from the available network outputs. In this letter, the network measurements are assumed to satisfy

$$
y(t)=C u(t)+f(t, u(t))
$$

where $y(t) \in \mathbb{R}^{m}$ is the measurement output, $C$ is a known constant matrix with appropriate dimension. $f: \mathbb{R} \times \mathbb{R}^{n} \rightarrow \mathbb{R}^{m}$ is the neurondependent nonlinear disturbances on the network outputs, and satisfies the following Lipschitz condition:

$$
|f(t, x)-f(t, y)| \leq|F(x-y)|
$$

where the constant matrix $F \in \mathbb{R}^{n \times n}$ is known. Note that the nonlinearity description given in (5) has been frequently used in many papers dealing with nonlinear analysis [16].

In this letter, the full-order state estimator is of the form

$$
\begin{aligned}
\dot{\hat{u}}(t)= & -A \hat{u}(t)+W_{0} g(\hat{u}(t))+W_{1} g(\hat{u}(t-h(t)))+V \\
& +K[y(t)-C \hat{u}(t)-f(t, \hat{u}(t))]
\end{aligned}
$$

where $\hat{u}(t)$ is the estimation of the neuron state, and $K \in \mathbb{R}^{n \times m}$ is the estimator gain matrix to be designed.

Let the error state be

$$
e(t)=u(t)-\hat{u}(t)
$$

then it follows from (1), (4), and (6) that:

$$
\begin{aligned}
\dot{e}(t)= & (-A-K C) e(t)+W_{0}[g(u(t))-g(\hat{u}(t))] \\
& +W_{1}[g(u(t-h(t)))-g(\hat{u}(t-h(t)))] \\
& -K[f(t, u(t))-f(t, \hat{u}(t))] .
\end{aligned}
$$

Now, let $e(t ; \xi)$ denote the state trajectory of the error-state system (8) from the initial data $e(\theta)=\xi(\theta)$ on $-h \leq \theta \leq 0$ in $L_{\mathcal{F}_{0}}^{2}\left([-h, 0] ; \mathbb{R}^{n}\right)$. It can be easily seen that the system (8) admits a trivial solution $e(t ; 0) \equiv 0$ corresponding to the initial data $\xi=0$.

Definition 1: For the system (8) and every $\xi \in L_{\mathcal{F}_{0}}^{2}\left([-h, 0] ; \mathbb{R}^{n}\right)$ where $h$ is the upper bound of the time-delay, the trivial solution is globally exponentially stable if there exist constants $\alpha>0$ and $\beta>0$ such that

$$
|e(t ; \xi)|^{2} \leq \alpha e^{-\beta t} \sup _{-h \leq \theta \leq 0}|\xi(\theta)|^{2}
$$

We shall design a state estimator for the delayed neural network described by (1) and (4), such that the dynamics of the system (8) is globally exponentially stable, for the nonlinear activation function $g(\cdot)$, the nonlinear disturbance $f(\cdot, \cdot)$, and the time-varying delay $h(t)$.

\section{MaIn Results AND PROOFS}

The following lemma, known as Schur Complement Lemma, will be essential in establishing our results in terms of LMIs.

Lemma 1: [3] Given constant matrices $\Omega_{1}, \Omega_{2}, \Omega_{3}$ where $\Omega_{1}=\Omega_{1}^{T}$ and $0<\Omega_{2}=\Omega_{2}^{T}$, then

$$
\Omega_{1}+\Omega_{3}^{T} \Omega_{2}^{-1} \Omega_{3}<0
$$

if and only if

$$
\left[\begin{array}{cc}
\Omega_{1} & \Omega_{3}^{T} \\
\Omega_{3} & -\Omega_{2}
\end{array}\right]<0, \quad \text { or } \quad\left[\begin{array}{cc}
-\Omega_{2} & \Omega_{3} \\
\Omega_{3}^{T} & \Omega_{1}
\end{array}\right]<0
$$

We first derive the conditions under which the error dynamics of the estimation process is globally exponentially stable. The following theorem shows that such conditions can be expressed in terms of the positive-definite solution to a quadratic matrix inequality involving several scalar parameters.

Theorem 1: Let the estimator gain $K$ be given. If there exist positive scalars $\varepsilon_{1}>0, \varepsilon_{2}>0, \varepsilon_{3}>0$ and a positive-definite matrix $P>0$ such that the following quadratic matrix inequality

$$
\begin{aligned}
& (-A-K C)^{T} P+P(-A-K C) \\
& +P\left(\varepsilon_{1}^{-1} W_{0} W_{0}^{T}+\varepsilon_{2}^{-1} W_{1} W_{1}^{T}+\varepsilon_{3}^{-1} K K^{T}\right) P \\
& +\varepsilon_{1} G^{T} G+\varepsilon_{2}(1-d)^{-1} G^{T} G+\varepsilon_{3} F^{T} F<0
\end{aligned}
$$

holds, then the error-state system (8) of the neural network is globally exponentially stable.

Proof: For notational simplicity, we define

$$
\begin{aligned}
A_{K} & :=-A-K C \\
\psi(t) & :=g(u(t))-g(\hat{u}(t)) \\
\phi(t) & :=f(t, u(t))-f(t, \hat{u}(t)) \\
\Psi & :=\left[\begin{array}{c}
\varepsilon_{1}^{1 / 2} \psi(t)-\varepsilon_{1}^{-1 / 2} W_{0}^{T} P e(t) \\
\varepsilon_{2}^{1 / 2} \psi(t-h(t))-\varepsilon_{2}^{-1 / 2} W_{1}^{T} P e(t) \\
\varepsilon_{3}^{1 / 2} \phi(t)+\varepsilon_{3}^{-1 / 2} K^{T} P e(t)
\end{array}\right] .
\end{aligned}
$$


From (3) and (5), we have immediately that

$$
\begin{aligned}
\psi^{T}(t) \psi(t):= & |g(u(t))-g(\hat{u}(t))|^{2} \\
& \leq|G e|^{2}=e^{T} G^{T} G e \\
\phi^{T}(t) \phi(t):= & |f(t, u(t))-f(t, \hat{u}(t))|^{2} \\
& \leq|F e|^{2}=e^{T} F^{T} F e .
\end{aligned}
$$

Following the similar line of the proof of [16, Th.1], using the facts $\Psi^{T} \Psi \geq 0,0 \leq \dot{h}(t) \leq d<1$ and the relations (10), (15), and (16), we can establish that

$$
\begin{gathered}
\frac{d \Phi(e(t))}{d t} \\
\leq e^{T}(t)\left\{A_{K}^{T} P+P A_{K}+P\left(\varepsilon_{1}^{-1} W_{0} W_{0}^{T}+\varepsilon_{2}^{-1} W_{1} W_{1}^{T}\right.\right. \\
\left.+\varepsilon_{3}^{-1} K K^{T}\right) P+\varepsilon_{1} G^{T} G \\
\left.+\varepsilon_{2}(1-d)^{-1} G^{T} G+\varepsilon_{3} F^{T} F\right\} e(t)<0
\end{gathered}
$$

where the Lyapunov functional candidate is given by

$$
\begin{aligned}
\Phi(e(t)) & =e^{T}(t) P e(t)+\int_{t-h(t)}^{t} e^{T}(s) Q e(s) d s \\
Q: & =\varepsilon_{2}(1-d)^{-1} G^{T} G
\end{aligned}
$$

and $P$ is the positive-definite solution to the inequality (10).

From Lyapunov stability theory, we arrive at the conclusion that the error-state system (8) is asymptotically stable. In order to prove the expected global exponential stability of the system (8), we will need to make some standard manipulations on the relation (17). The detailed proof follows similar line of the proof of [16, Th. 1] and is, thus, omitted. We just mention here that in the Definition 1 , the constant $\beta$ is the unique positive root of the equation

$$
\lambda_{\min }(-\Pi)-\beta \lambda_{\max }(P)-\beta h \lambda_{\max }(Q) e^{\beta h}=0
$$

where

$$
\begin{array}{rl}
\Pi:=A_{K}^{T} & P+P A_{K}+P\left(\varepsilon_{1}^{-1} W_{0} W_{0}^{T}+\varepsilon_{2}^{-1} W_{1} W_{1}^{T}\right. \\
& \left.+\varepsilon_{3}^{-1} K K^{T}\right) P+\varepsilon_{1} G^{T} G+\varepsilon_{2}(1-d)^{-1} G^{T} G+\varepsilon_{3} F^{T} F
\end{array}
$$

where $h(0 \leq h(t) \leq h)$ is the maximum of the time-varying state delay. Another constant $\alpha$ is determined by

$$
\alpha=\lambda_{\min }^{-1}(p)\left[\lambda_{\max }(p)+h \lambda_{\max }(Q)\left(1+\beta h e^{\beta h}\right)\right] .
$$

This completes the proof of Theorem 1.

In Theorem 1, when an estimator is given, the analysis result (i.e., the stability criterion) is established in terms of a quadratic matrix equality (10), which contains several scalar parameters. Next, we deal with the corresponding design problem, which aims to design the estimator gain, $K$, such that (10) holds for positive scalars $\varepsilon_{1}>0, \varepsilon_{2}>0$, $\varepsilon_{3}>0$ and a positive-definite matrix $P>0$.

It should be mentioned that, in the past decade, linear matrix inequalities (LMIs) have gained much attention for their computational tractability and usefulness in many areas, including the stability testing for neural networks [12], because the so-called interior point method [3] has been proven to be numerically very efficient for solving the LMIs. Thus, our next goal is to develop an LMI approach to designing the desired estimator gains for the delayed neural networks.

Theorem 2: If there exist three positive scalars $\varepsilon_{1}, \varepsilon_{2}, \varepsilon_{3}$, a positive-definite matrix $P \in \mathbb{R}^{n \times n}$ and a matrix $R \in \mathbb{R}^{n \times n}$ such that the linear matrix inequality, shown in (19) at the bottom of the page, holds, then with the estimator gain

$$
K=P^{-1} R
$$

the error-state system (8) of the delayed neural network described by (1) and (4) is globally exponentially stable.

Proof: First of all, note that $A$ is a diagonal matrix, hence, $A^{T}=$ $A$. Pre- and postmultiplying the inequality (19) by the block-diagonal matrix

$$
\operatorname{diag}\left\{I, \varepsilon_{1}^{-\frac{1}{2}} I, \varepsilon_{1}^{-\frac{1}{2}} I, \varepsilon_{2}^{-\frac{1}{2}} I, \varepsilon_{2}^{-\frac{1}{2}} I, \varepsilon_{3}^{-\frac{1}{2}} I, \varepsilon_{3}^{-\frac{1}{2}} I\right\}
$$

yield (21), shown at the bottom of the page, or

$$
\left[\begin{array}{cc}
\Omega_{1} & \Omega_{3}^{T} \\
\Omega_{3} & -\Omega_{2}
\end{array}\right]<0
$$

$$
\left[\begin{array}{ccccccc}
-A P-P A-C^{T} R^{T}-R C & P W_{0} & \varepsilon_{1} G^{T} & P W_{1} & \varepsilon_{2}(1-d)^{-\frac{1}{2}} G^{T} & R & \varepsilon_{3} F^{T} \\
W_{0}^{T} P & -\varepsilon_{1} I & 0 & 0 & 0 & 0 & 0 \\
\varepsilon_{1} G & 0 & -\varepsilon_{1} I & 0 & 0 & 0 & 0 \\
W_{1}^{T} P & 0 & 0 & -\varepsilon_{2} I & 0 & 0 & 0 \\
\varepsilon_{2}(1-d)^{-\frac{1}{2}} G & 0 & 0 & 0 & -\varepsilon_{2} I & 0 & 0 \\
R^{T} & 0 & 0 & 0 & 0 & -\varepsilon_{3} I & 0 \\
\varepsilon_{3} F & 0 & 0 & 0 & 0 & 0 & -\varepsilon_{3} I
\end{array}\right]<0
$$

$$
\left[\begin{array}{ccccccc}
-A P-P A-C^{T} R^{T}-R C & \varepsilon_{1}^{-\frac{1}{2}} P W_{0} & \varepsilon_{1}^{\frac{1}{2}} G^{T} & \varepsilon_{2}^{-\frac{1}{2}} P W_{1} & \varepsilon_{2}^{\frac{1}{2}}(1-d)^{-\frac{1}{2}} G^{T} & \varepsilon_{3}^{-\frac{1}{2}} R & \varepsilon_{3}^{\frac{1}{2}} F^{T} \\
\varepsilon_{1}^{-\frac{1}{2}} W_{0}^{T} P & -I & 0 & 0 & 0 & 0 & 0 \\
\varepsilon_{1}^{\frac{1}{2}} G & 0 & -I & 0 & 0 & 0 & 0 \\
\varepsilon_{2}^{-\frac{1}{2}} W_{1}^{T} P & 0 & 0 & -I & 0 & 0 & 0 \\
\varepsilon_{2}^{\frac{1}{2}}(1-d)^{-\frac{1}{2}} G & 0 & 0 & 0 & -I & 0 & 0 \\
\varepsilon_{3}^{-\frac{1}{2}} R^{T} & 0 & 0 & 0 & 0 & -I & 0 \\
\varepsilon_{3}^{\frac{1}{2}} F & 0 & 0 & 0 & 0 & 0 & -I
\end{array}\right]
$$


where

$$
\begin{aligned}
\Omega_{1} & :=-A P-P A-C^{T} R^{T}-R C \\
\Omega_{2} & :=I \\
\Omega_{3} & :=\left[\begin{array}{llll}
\varepsilon_{1}^{-\frac{1}{2}} P W_{0} & \varepsilon_{1}^{\frac{1}{2}} G^{T} & \varepsilon_{2}^{-\frac{1}{2}} P W_{1} \\
& \varepsilon_{2}^{\frac{1}{2}}(1-d)^{-\frac{1}{2}} G^{T} & \varepsilon_{3}^{-\frac{1}{2}} R & \varepsilon_{3}^{\frac{1}{2}} F^{T}
\end{array}\right]^{T} .
\end{aligned}
$$

It follows from the Schur Complement Lemma (Lemma 1) that (22) holds if and only if:

$$
\Omega_{1}+\Omega_{3}^{T} \Omega_{2}^{-1} \Omega_{3}<0
$$

or

$$
\begin{aligned}
- & A P-P A-C^{T} R^{T}-R C+\varepsilon_{1}^{-1} P W_{0} W_{0}^{T} P \\
& +\varepsilon_{1} G^{T} G+\varepsilon_{2}^{-1} P W_{1} W_{1}^{T} P+\varepsilon_{2}(1-d)^{-1} G^{T} G \\
& +\varepsilon_{3}^{-1} P K K^{T} P+\varepsilon_{3} F^{T} F<0 .
\end{aligned}
$$

Noticing that $R=P K$, it can be easily seen that (23) is the same as (21). Hence, it follows from Theorem 1 that, with the estimator gain given by (20), the error-state system (8) of the delayed neural network described by (1) and (4) is globally exponentially stable. This ends the proof of Theorem 2.

Remark 1: Notice that in Theorem 2, the matrix inequality (21) is linear on the parameters $\varepsilon_{1}>0, \varepsilon_{2}>0, \varepsilon_{3}>0, P>0$, and $Q$. Therefore, by using the Matlab LMI toolbox, it is straightforward to design the neuron state estimator which guarantees the global exponential convergence of the error dynamics. Furthermore, if the delay is constant [1], [9], [10], [15], [17], we just need to let $d=0$ in our main results.

\section{A Special CASE}

In the literature, a vast amount of results have been published on the general topic of the stability analysis of the delayed neural networks. Various issues have been investigated, such as the asymptotic stability case or the exponential stability case, the constant delay case or the time-varying delay case, the delay-independent stability criteria or the delay-dependent stability criteria, etc., see [1], [4], [10]-[12], and [17] for some examples. It should be pointed out that, although this letter is focused on the neuron estimation problem, within the same framework, the main results can still be generalized to the conventional stability analysis problem for delayed neural networks.

Consider the delayed neural network (1), and let $u^{*}$ be its equilibrium point. For presentation convenience, we can shift the intended equilibrium $u^{*}$ to the origin by letting $x=u-u^{*}$, and then the system (1) can be transformed into

$$
\begin{aligned}
& \dot{x}(t)=-A x(t)+W_{0} l(x(t))+W_{1} l(x(t-h(t))) \\
& x(t)=\varphi(t), \quad t \in[-h, 0], \quad h=\sup _{t \in[0, \infty)} h(t)
\end{aligned}
$$

where $x(t)=\left[x_{1}(t), x_{2}(t), \cdots, x_{n}(t)\right]^{T} \in \mathbb{R}^{n}$ is the state vector of the transformed system, and the transformed neuron activation function $l(x)=g\left(x+u^{*}\right)-g\left(u^{*}\right)$ satisfies

$$
|l(x)| \leq|M x|
$$

where $M \in \mathbb{R}^{n \times n}$ is a known constant matrix. Notice that we do not need the traditional monotonicity and smoothness assumptions on the activation function $l(x)$.
The stability analysis problem for the delayed neural network of the type (24) has attracted significant research interests [1], [4], [10]-[12]. Our results derived in the previous section can be used to develop an alternative criterion for testing the exponential stability of the neural network (29).

Theorem 3: If there exist positive scalars $\varepsilon_{1}, \varepsilon_{2}$ and a positive-definite matrix $P \in \mathbb{R}^{n \times n}$ such that the following linear matrix inequality:

$$
\left[\begin{array}{ccccc}
-A P-P A & P W_{0} & \varepsilon_{1} M^{T} & P W_{1} & \varepsilon_{2}(1-d)^{-\frac{1}{2}} M^{T} \\
W_{0}^{T} P & -\varepsilon_{1} I & 0 & 0 & 0 \\
\varepsilon_{1} M & 0 & -\varepsilon_{1} I & 0 & 0 \\
W_{1}^{T} P & 0 & 0 & -\varepsilon_{2} I & 0 \\
\varepsilon_{2}(1-d)^{-\frac{1}{2}} M & 0 & 0 & 0 & -\varepsilon_{2} I
\end{array}\right]<0
$$

holds, then the delayed neural network (24) is globally exponentially stable.

Proof: The proof follows the same line of the proofs of Theorem 1 and Theorem 2 and is, thus, omitted.

Remark 2: Theorem 3 reveals that the exponential stability of the delayed network (24) can be checked by examining the solvability of the LMI (24), which can be readily conducted by utilizing the Matlab LMI toolbox. In contrast to the existing results on delay-independent asymptotical stability, such as those given in [1], [4], our LMI approach has the advantage that the LMIs can be solved numerically and effectively by using the interior-point method [3].

\section{NUMERICAL EXAMPLES}

Two simple examples are presented here so as to illustrate the usefulness of our main results.

Example 1: In this example, we examine the exponential stability of a delayed neural network.

Consider a two-neuron neural network (24), where

$$
\begin{aligned}
A & =\left[\begin{array}{cc}
0.8 & 0 \\
0 & 0.8
\end{array}\right] W_{0}=\left[\begin{array}{cc}
1 & -2 \\
-2 & 1
\end{array}\right] W_{1}=\left[\begin{array}{ll}
1 & 1 \\
1 & 1
\end{array}\right] \\
M & =\left[\begin{array}{cc}
0.1 & 0 \\
0 & 0.1
\end{array}\right] d=0.1 .
\end{aligned}
$$

By using the Matlab LMI toolbox, we solve the LMI (26) for $\varepsilon_{1}>0$, $\varepsilon_{2}>0, P>0$ and obtain

$$
\varepsilon_{1}=12.3045 \quad \varepsilon_{2}=15.9232 \quad P=\left[\begin{array}{ll}
1.1342 & 0.0467 \\
0.0467 & 1.3459
\end{array}\right]
$$

Therefore, it follows from Theorem 3 that the two-neuron neural network (24) is globally exponentially stable. The response of the state dynamics to the initial condition $(10,3)$ is shown in Fig. 1, which verifies our theoretical conclusion.

Example 2: Now, we demonstrate how to design an estimator for the delayed neural network. Assume that the delayed neural network in (1) and (4) is described by the following data:

$$
\begin{aligned}
A & =\left[\begin{array}{lll}
2 & 0 & 0 \\
0 & 3 & 0 \\
0 & 0 & 2
\end{array}\right] \quad W_{0}=\left[\begin{array}{ccc}
0.2 & -0.4 & 0.4 \\
-0.4 & 0.2 & 0.2 \\
0.2 & 0.4 & -0.4
\end{array}\right] \\
W_{1} & =\left[\begin{array}{lll}
0.2 & 0.2 & 0.2 \\
0.2 & 0.2 & 0.2 \\
0.2 & 0.2 & 0.2
\end{array}\right] \quad V=\left[\begin{array}{lll}
0.5 & 0.5 & 0.5
\end{array}\right]^{T} \\
C & =I_{3} \quad h(t)=0.4 \sin (t)(d=0.4) \\
G & =0.5 I_{3} \quad F=0.4 I_{3} .
\end{aligned}
$$




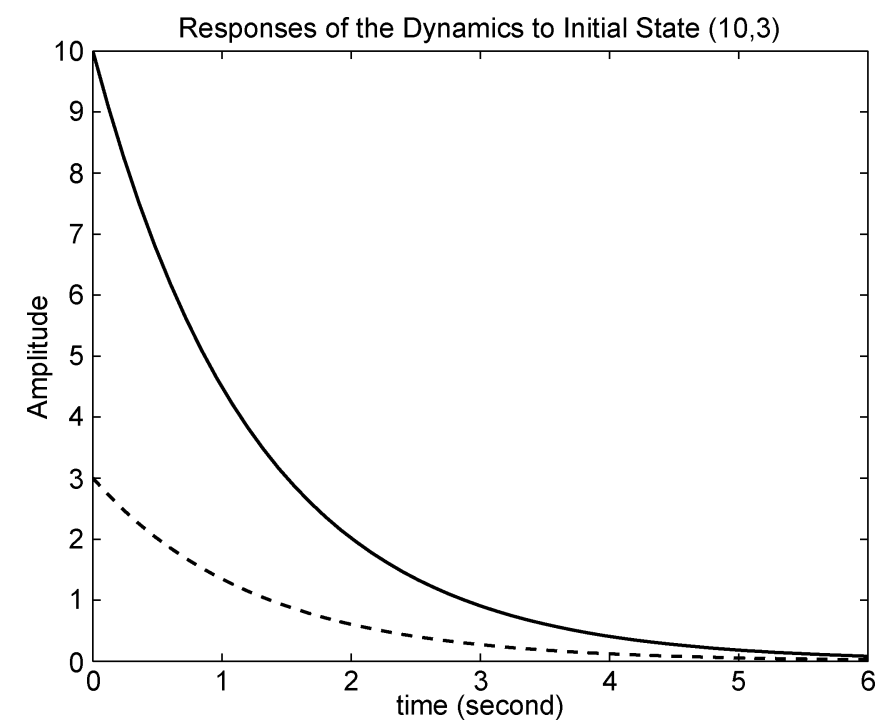

Fig. 1. $x_{1}$ (solid), $x_{2}$ (dashed).

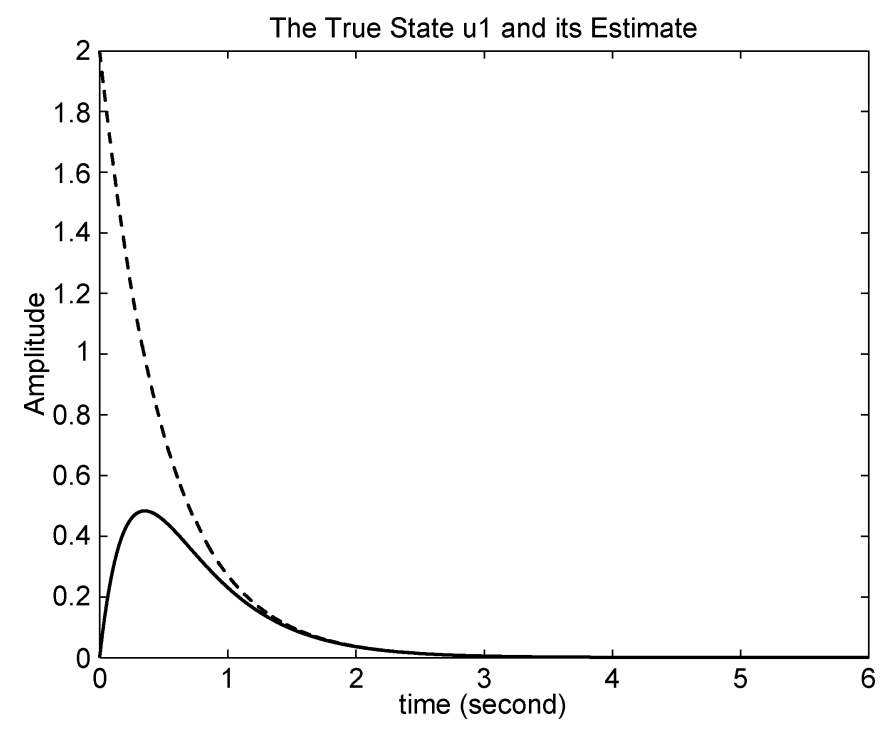

Fig. 2. $\quad u_{1}$ (dashed), $\hat{u}_{1}$ (solid).

Solving the LMI (19) for $\varepsilon_{1}>0, \varepsilon_{2}>0, \varepsilon_{3}>0, P>0$ and $R$ gives

$$
\begin{aligned}
& \varepsilon_{1}=1.3156 \quad \varepsilon_{2}=1.1134 \quad \varepsilon_{3}=0.9965 \\
& P=\left[\begin{array}{ccc}
1.3086 & 0.0003 & -0.0002 \\
0.0003 & 2.4436 & 0.0004 \\
-0.0002 & 0.0004 & 1.3077
\end{array}\right] \\
& R=\left[\begin{array}{ccc}
2.4939 & 0 & 0 \\
0 & -0.5009 & 0 \\
0 & 0 & -0.5009
\end{array}\right]
\end{aligned}
$$

and, hence, we have

$$
K=P^{-1} R=\left[\begin{array}{ccc}
1.9058 & 0.0000 & -0.0001 \\
-0.0002 & -0.2050 & 0.0001 \\
0.0003 & 0.0001 & -0.3830
\end{array}\right]
$$

The true state $u_{1}$ (respectively, $u_{2}, u_{3}$ ) and its estimate $\hat{u}_{1}$ (respectively, $\hat{u}_{2}, \hat{u}_{3}$ ) are displayed in Fig. 2 (Figs. 3 and 4, respectively). The simulation results imply that, with the obtained estimator gain $K$, the error dynamics for the neural network converges to zero exponentially.

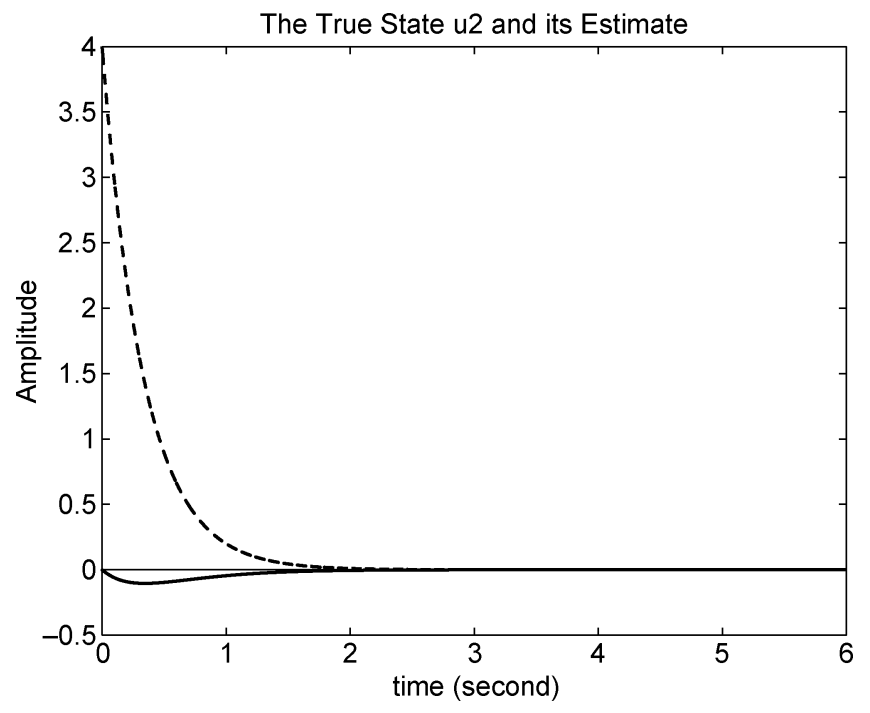

Fig. 3. $u_{2}$ (dashed), $\hat{u}_{2}$ (solid).

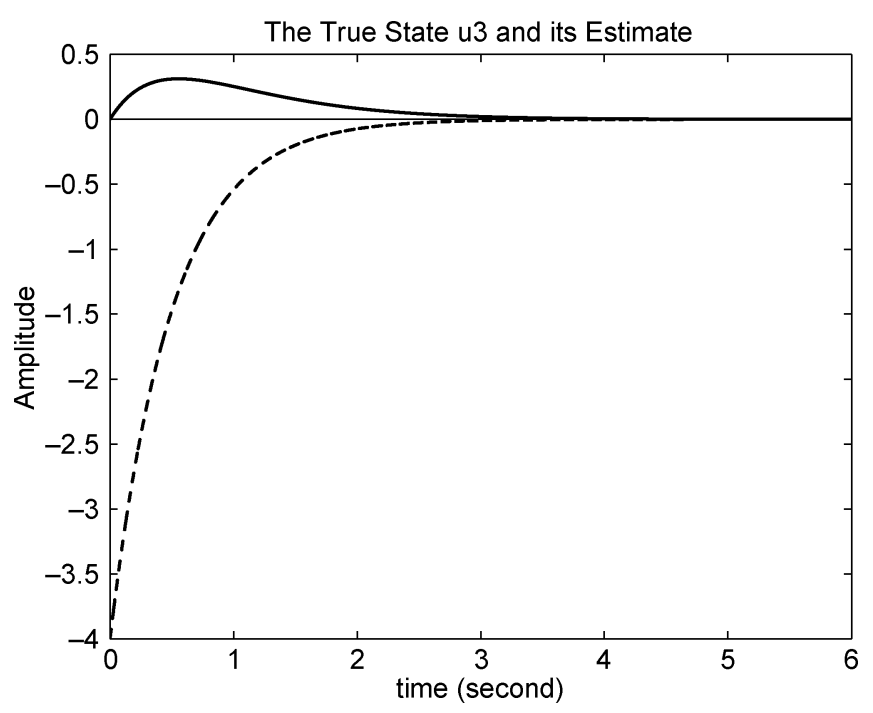

Fig. 4. $u_{3}$ (dashed), $\hat{u}_{3}$ (solid).

In other words, the neuron states are tracked very well by the designed estimator.

\section{CONCLUSION}

In this letter, we have dealt with the problem of state estimation for a class of delayed neural networks. We have removed the traditional monotonicity and smoothness assumptions on the activation function. A linear matrix inequality (LMI) approach has been developed to solve the problem addressed. Specifically, the conditions for the existence of the expected estimators have been derived in terms of the positive-definite solution to an LMI involving several scalar parameters, and the analytical expression characterizing the desired estimators has been obtained. We have also shown that the main results can be easily extended to cope with the stability analysis problem for delayed neural networks. Finally, two numerical examples have been used to demonstrate the usefulness of the main results.

\section{ACKNOWLEDGMENT}

Z. Wang would like to thank Prof. H. Unbehauen, Ruhr-University Bochum, Germany, for detailed comments, and Prof. D. Prätzel-Wolters, University of Kaiserslautern, Germany, for helpful suggestions. 


\section{REFERENCES}

[1] S. Arik, "Stability analysis of delayed neural networks," IEEE Tran. Circuits Syst. I, vol. 47, no. 7, pp. 1089-1092, Jul. 2000.

[2] P. Baldi and A. F. Atiya, "How delays affect neural dynamics and learning," IEEE Trans. Neural Netw., vol. 5, no. 4, pp. 612-621, Jul. 1994.

[3] S. Boyd, L. E. I. Ghaoui, E. Feron, and V. Balakrishnan, Linear Matrix Inequalities in System and Control Theory. Philadephia, PA: SIAM, 1994.

[4] J. Cao, "Periodic oscillation and exponential stability of delayed CNNs," Phys. Lett. A, vol. 270, pp. 157-163, 2000.

[5] M. A. Cohen and S. Grossberg, "Absolute stability and global pattern formation and parallel memory storage by competitive neural networks," IEEE Trans. Syst., Man, Cybern., vol. SMC-13, no. 5, pp. 815-821, Oct. 1983.

[6] V. T. S. Elanayar and Y. C. Shin, "Approximation and estimation of nonlinear stochastic dynamic systems using radial basis function neural networks," IEEE Trans. Neural Netw., vol. 5, no. 4, pp. 594-603, Jul. 1994.

[7] R. Habtom and L. Litz, "Estimation of unmeasured inputs using recurrent neural networks and the extended Kalman filter," in Proc. Int. Conf. Neural Networks, vol. 4, Houston, TX, 1997, pp. 2067-2071.

[8] J. K. Hale and S. M. V. Lunel, "Introduction to the theory of functional differential equations," in Applied Mathematical Sciences. New York: Springer-Verlag, 1991, vol. 99.

[9] M. P. Joy, "On the global convergence of a class of functional differential equations with applications in neural network theory," J. Math. Anal. Applicat., vol. 232, pp. 61-81, 1999.
[10] - "Results concerning the absolute stability of delayed neural networks," Neural Netw., vol. 13, pp. 613-616, 2000.

[11] X. F. Liao, K. W. Wong, C. S. Leung, and Z. F. Wu, "Hopf bifurcation and chaos in a single delayed neuron equation with nonmonotonic activation function," Chaos Solitons Fractals, vol. 12, pp. 1535-1547, 2001.

[12] X. F. Liao, G. Chen, and E. N. Sanchez, "LMI-based approach for asymptotically stability analysis of delayed neural networks," IEEE Transactions on Circuits Systems-I, vol. 49, no. 7, pp. 1033-1039, Jul. 2002.

[13] M. Morita, "Associative memory with nonmonotone dynamics," Neural Netw., vol. 6, no. 6, pp. 115-126, 1993.

[14] F. M. Salam and J. Zhang, "Adaptive neural observer with forward co-state propagation," in Proc. Int. Joint Conf. Neural Networks (IJCNN '01), vol. 1, Washington, DC, 2001, pp. 675-680.

[15] P. Van den Driessche and X. Zou, "Global attractivity in delayed Hopfield neural networks model," SIAM J. Appl. Math., vol. 58, pp. 1878-1890, 1998.

[16] Z. Wang, D. P. Goodall, and K. Burnham, "On designing observers for time-delay systems with nonlinear disturbances," Int. J. Control, vol. 75, pp. 803-811, 2002.

[17] Y. Zhang, "Global exponential stability and periodic solutions of delay Hopfield neural networks," Int. J. Syst. Sci., vol. 27, pp. 227-231, 1996.

[18] Y. Zhang, P. A. Heng, and A. W. C. Fu, "Estimate of exponential convergence rate and exponential stability for neural networks," IEEE Trans. Neural Netw., vol. 10, no. 6, pp. 1487-1493, Nov. 1999. 